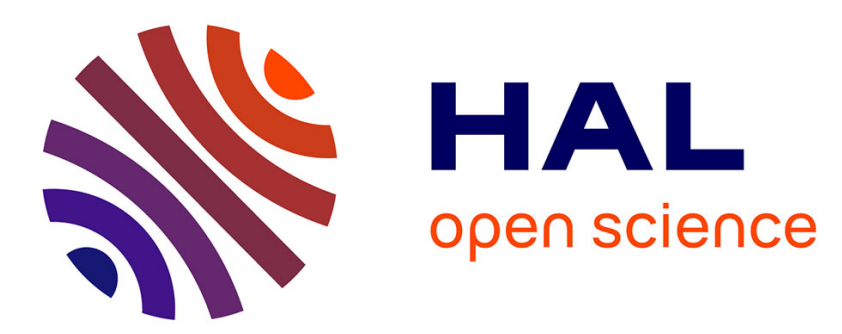

\title{
Les plans d'aménagement ou plans de gestion adaptés aux conditions particulières de la forêt privée
}

\author{
Louis Bourgenot
}

\section{To cite this version:}

Louis Bourgenot. Les plans d'aménagement ou plans de gestion adaptés aux conditions particulières de la forêt privée. Revue forestière française, 1963, 8-9, pp.675-681. 10.4267/2042/24575 . hal-03389828

\section{HAL Id: hal-03389828 \\ https://hal.science/hal-03389828}

Submitted on 21 Oct 2021

HAL is a multi-disciplinary open access archive for the deposit and dissemination of scientific research documents, whether they are published or not. The documents may come from teaching and research institutions in France or abroad, or from public or private research centers.
L'archive ouverte pluridisciplinaire HAL, est destinée au dépôt et à la diffusion de documents scientifiques de niveau recherche, publiés ou non, émanant des établissements d'enseignement et de recherche français ou étrangers, des laboratoires publics ou privés. 


\section{LES PLANS D'AMÉNAGEMENT OU PLANS DE GESTION ADAPTES AUX CONDITIONS PARTICULIËRES DE LA FORÊT PRIVÉE}

PAR

\section{BOURGENOT}

Chef du service des forêts soumises au régime forestier

I1 me semble indispensable, avant de parler d'aménagement de la forêt, de rassurer le lecteur sur cette notion qu'à notre époque, pourtant placée sous le signe de la planification, beaucoup assimilent encore à un jeu de l'esprit, d'une complexité rebutante, d'une nécessité plus que douteuse, et que la tradition imposerait aux forêts domaniales, mais qui serait inutile en forêt privée.

I1 ne viendrait à l'idée de personne - et celle du facteur Cheval, dans la Drôme, est l'exception qui confirme la règle, - de construire sa maison sans un plan; de même, aucun industriel ne songerait à mettre en œuvre un accroissement de sa production, ou, à fortiori, à une reconversion de son entreprise après réflexion sur cette production, sans avoir étudié au préalable les conditions du marché, la progression des investissements nécessaires, les prix de revient, en un mot sans un programme raisonné de développement fondé sur l'étude du bilan économique et financier des opérations projetées.

Toutes proportions gardées, l'aménagement, pour une forêt, est l'homologue du plan pour une maison, du programme de développement pour une industrie. Mais il faut immédiatement ajouter une restriction importante. La forêt est en effet un organisme vivant complexe, et son aménagement prétend planifier dans un domaine beaucoup plus difficile à appréhender que celui de l'acier et du béton, car il s'agit d'un domaine aléatoire de par l'indétermination qui 
règne à la fois sur les conditions de croissance des arbres et des peuplements, et sur le parti économique que l'on pourra en tirer dans l'avenir.

Pourquoi, dans ces conditions, me direz-vous, cette manie de la planification? - Puisque les prévisions sont ici si difficiles, pourquoi s'acharner à prévoir?

Deux exemples vaudront mieux, en guise de réponse, que de longs discours.

\section{Premier exemple:}

Le premier exemple sera celui d'un taillis-sous-futaie que votre grand-père, puis votre père se sont ingéniés à enrichir par des martelages prudents. Vous vous trouvez aujourd'hui en possession d'une sorte de futaie, certes assez claire, mais relativement riche en gros bois, et vous ne parlez plus du taillis que pour mémoire car il n'a plus de valeur. Pour mémoire, dans vos supputations financières certes, mais non dans vos préoccupations d'ordre sylvicole, car il est̆ bien gênant parfois et, tous comptes faits, votre forêt serait bien plus facile à gérer s'il n'existait pas.

Voire. Car vous avez bien l'intention de continuer à vendre une coupe tous les ans. Taillis ou pas, comment allez-vous la marquer? Ce capital de gros bois accumulé par les générations qui vous ont précédé et par vous-même, il vous semble raisonnable, sans cesser de traiter votre forêt en bon père de famille, de commencer à l'entamer. D'ailleurs, pensez-vous, à juste raison, les chênes ni les hêtres ne sont éternels, et je me vois dans l'obligation, chaque année, de récolter des quantités de plus en plus importantes d'arbres mûrs, donc de gros diamètres, donc de grosse valeur. Et tant mieux pour moi, et grands mercis à mes ä̈eux prévoyants.

Mais pouvez-vous vous contenter d'arrêter là votre raisonnement? Si oui, il y a fort à parier que votre fils ou votre petitfils ne se trouvent, eux, en présence d'un taillis simple de charme, le capital accumulé pendant plusieurs générations se trouvant, par la force des choses, éliminé par la dernière, sans aucun renouvellement, comme c'est le cas très général dans les taillis-sous-futaie enrichis.

A moins bien sûr, que vous décidiez de vous arrêter à cet “ Après moi le déluge "), vous voilà obligé, si vous avez le double souci de votre postérité et de votre titre de propriétaire forestier sylviculteur, de vous pencher, que vous le vouliez ou non, sur l'avenir de votre forêt. Le résultat de vos réflexions ne fait pas de doute: vous ne pouvez plus vous permettre d'exploiter de gros bois sans assurer un renouvellement de capital, c'est-à-dire sans prévoir le rajeunissement des peuplements en essences capables, plus tard, de procurer à vos enfants et petits-enfants un revenu au moins égal au vôtre actuel. 
Sans pour autant acheminer ces réflexions dans les sentiers ténébreux de doctrines ésotériques, vous comprendrez ensuite très vite que pour assurer ce rajeunissement, vous disposez d'un temps limité: tout longévifs qu'ils soient, les arbres ne peuvent fructifier et fabriquer de matériaux sains que jusqu'à un certain âge $\mathrm{A}$; si, dans l'ensemble ils sont actuellement âgés de B, ce temps limité n'est autre que $A-B$. Vous avez donc $A-B$ années devant vous pour régénérer votre forêt.

Mais que faites-vous? Comme M. Jourdain, de la prose, de l'aménagement, sans le savoir.

Car vos réflexions ne peuvent s'arrêter à ce stade, où nous allons retrouver, mutatis mutandis, le même genre de problèmes, que ceux qu'ont à résoudre le constructeur d'une maison ou l'industriel évoqués ci-dessus.

En quelles essences avez-vous intérêt à régénérer votre forêt? Devez-vous vous arrêter au chêne et au hêtre spontanés, ou à d'autres espèces? Dans ce dernier cas, quelles espèces pouvez-vous envisager compte tenu des conditions locales (sol, climat, etc...) et de leur valeur technologique? Quel rythme de régénération adopter, quelle répartition dans le temps des travaux que cette régénération entraînera, et donc des investissements à consentir?

Aménager votre forêt, ce n'est pas autre chose que répondre à ces questions; et c'est dans la mesure où ces questions et les réponses à leur donner vous sembleront importantes que l'anénagement vous apparaitra comme un acte nécessaire. Le départ entre nécessité et inutilité de l'aménagement se situe donc exactement à la limite entre les deux propositions suivantes: ou bien vous prenez conscience de la nécessité, pour vous-même ou pour vos descendants, de prendre une possession complète de votre forêt, comme le dit le professeur L. Schaeffer, c'est-à-dire de savoir le pourquoi et le comment de vos interventions, ou bien vous désirez marquer vos coupes sinon au petit bonheur, du moins à l'estime, sans vous inquiéter de l'avenir des peuplements et de leur production.

Permettez-moi d'insister en passant sur la définition de $L$. Schaeffer. Prendre une possession complète de la forêt, c'est projeter dans l'avenir la conséquence de toutes les décisions que vous êtes amené à prendre dans l'immédiat. Vous ne pouvez donc pas dire, par exemple: “ exécutons d'abord les travaux les plus urgents, marquons d'abord les gros bois, exécutons d'abord telle ou telle plantation, nous aménagerons par la suite ). C'est en marquant la coupe ou en plantant qu'il faut aménager; plus exactement, c'est avant: c'est avant d'exécuter qu'il faut choisir.

Il y a en effet un choix à faire, entre le " petit bonheur ) et la réflexion sur l'avenir. Ce qu'il faut savoir, c'est que dans le premier cas, vous risquez, au pire, de ruiner votre forêt, - que vos coupes soient trop fortes ou trop faibles -, au mieux, de la trans- 
former en une juxtaposition de peuplements hétéroclites, ce qui entrainera une commercialisation difficile des coupes, et non équilibrés, ce qui entraînera des à-coups dans la production.

\section{Second exemple:}

Je viens d'écrire " que vos coupes soient trop fortes ou trop faibles ». Permettez-moi d'expliquer ce paradoxe apparent sur un second exemple, que je choisirai très différent du premier. Ce sera une sapinière jardinée, par exemple d'une centaine d'hectares, que je supposerai en bon état et convenablement pourvue de jeunesse. Très sagement, vous l'avez partagée en dix divisions, et vous la parcourez à raison d'une division par an; c'est déjà l'excellente amorce d'un aménagement.

Mais avez-vous pensé à la quotité des coupes? Si celles que vous marquez sont trop fortes, vous ruinez insensiblement votre forêt; si elles sont trop faibles, vous y accumulez insensiblement un matériel de plus en plus élevé certes, mais comme la condition d'arbre est, comme la condition humaine, de naitre, de croître, de vieillir et de mourir, vous cultivez une forêt de plus en plus riche ent vieux bois d'où la jeunesse disparait, et dont l'avenir est de plus en plus inquiétant: ce sera la maturité, puis le dépérissement par grandes masses, et les années de vaches maigres après les années de vaches grasses.

Si donc (et, bien entendu, dans cette seule mesure), vous désirez épargner à vos descendants de telles catastrophes, vous êtes amené, que vous le vouliez ou non, d'une part, à déterminer le volume optimum à couper annuellement dans votre forêt, compte tenu de son état actuel et de son évolution souhaitable dans l'avenir, d'autre part à vous préoccuper de la proportion existante de semis et jeunes bois, et, au cas où elle vous apparaîtrait manifestement insuffisante, à envisager les travaux (dégagements, plantations) nécessaires pour rétablir la situation.

\section{L'aménagement:}

De ces considérations, qui ont été jusqu'ici présentées en ordre dispersé, il ressort que l'aménagement est une nécessité pour tous. ceux qui désirent posséder puis transmettre à leurs descendants une forêt en bon état, susceptible de fournir avec le minimum d'àcoups, le maximum de produits les plus recherchés, donc les plus rémunérateurs.

Dans cette optique, aménager une forêt c'est:

- d'abord fixer l'objectif à atteindre ;

- ensuite, déterminer l'ensemble des mesures nécessaires pour atteindre cet objectif, c'est-à-dire se proposer un plan de gestion. 


\section{L'objectif:}

Le propriétaire forestier doit d'abord, comme le constructeur ou comme l'industriel, se fixer un objectif quant à l'utilisation de sa forêt. Il peut par exemple désirer la conserver comme " espace vert », comme lieu de promenade, ou comme territoire de chasse. Très généralement, il en attendra plutôt des revenus annuels et désirera alors lui faire rendre le maximum.

Dans ce dernier cas, l'objectif ne peut être fixé arbitrairement; il dépend en effet d'un ensemble de sujétions qui imposent le choix entre un petit nombre de solutions:

- les conditions écologiques (sol, climat, présence du gibier, dangers d'incendie, etc...);

- les conditions économiques (tendances du marché du bois);

- l'état actuel de la forêt;

- les possibilités financières du propriétaire et la rentabilité des investissements éventuels à consentir.

La forêt étant située sur un sol de nature et de fertilité déterminées, dans un climat déterminé, on n'y pourra cultiver qu'un nombre réduit d'essences que l'on choisira parmi celles qui présentent actuellement un intérêt éconornique certain, ou dont les débouchés semblent devoir manifester une tendance à l'expansion.

Dans le premier exemple cité ci-dessus, si le taillis-sous-futaie est riche en essences feuillues spontanées productrices de matériaux de qualité (chêne de tranchage ou d'ébénisterie, hêtre à cœur blanc et à grain fin), l'on pourra poursuivre sa conversion en futaie feuillue. Si, au contraire, il s'agit d'une forêt ruinée constituée de taillis simple, ou de taillis-sous-futaie producteurs de chênes et de hêtres médiocres, on s'orientera vers une substitution d'essences (résineux, peupliers, etc...), les essences à introduire ayant fait leurs preuves, non seulement d'adaptation au sol et au climat considérés, et de bonne croissance, mais aussi de bonne commercialisation.

Dans le second exemple (sapinière), le choix de l'objectif n'a pas à donner lieu à discussion: une gestion raisonnée permettra de retirer de la forêt la production maxima, en quantité et en qualité, que permettent les conditions de sol et de climat.

\section{Le plan de gestion:}

Le plan de gestion est l'ensemble des mesures nécessaires, dans un temps déterminé, pour, compte tenu des moyens dont on dispose, atteindre l'objectif fixé, ou orienter la forêt dans la voie choisie.

Il faut noter d'abord que s'il est nécessaire, comme nous l'avons $\mathrm{vu}$, de tenter une planification à long terme de manière à éviter, par exemple, de ruiner la forêt ou d'y cultiver trop longtemps des bois surannés, cette planification ne peut présenter qu'un caractère indicatif; soumise à de nombreux aléas, la forêt se laisse en effet 
difficilement encadrer dans des prévisions trop rigides, et il est bon de faire le point à intervalles fréquents, ce dernier terme étant entendu à l'échelle de la vie des peuplements forestiers.

L'expérience montre que ces mises au point sont utiles tous les dix ans et deviennent nécessaires tous les vingt ans: ce sont des ordres de grandeur que l'on pourra adopter comme durée raisonnable d'application du plan de gestion.

Il faut ensuite remarquer qu'en règle très générale, on ne pourra obtenir le résultat cherché sans consentir à certaines dépenses, correspondant soit à des travaux d'entretien des peuplements (dégagements, nettoiements, plantations de complément; etc...), ou de l'infrastructure du massif (routes, assainissements, etc...), soit à des travaux d'équipement; notamment, l'influence du réseau routier sur la commercialisation des coupes est trop connue pour qu'il soit nécessaire d'y insister: il ne servirait à rien de prévoir à l'avance tout un programme de coupes si l'absence de voies de vidange devait en rendre la vente problématique.

Bien entendu, le montant annuel des sommes à réserver pour ces travaiux est fonction à la fois du revenu actuel de la forêt et des, possibilités financières du propriétaire. Toutefois, il faut bien comprendre que, compte tenu du souci justifié que ce dernier doit avoir de leur rentabilité, la productivité actuelle et future de la forêt ne peut être maintenue ou augmentée si l'on se contente d'y récolter des produits et d'en encaisser la valeur, sans aucun réinvestissement.

Le plan de gestion - et l'on m'excusera d'insister sur ce point important - n'est donc pas seulement une prévision de coupes mais aussi une prévision de travaux à échelonner rationnellement dans le temps, les investissements annuels à consentir devant être évalués après établissement d'un bilan économique et financier comme en toute entreprise.

Quant aux exploitations, le plan de gestion doit en prévoir notamment:

- la nature (régénération, éclaircie, jardinage, nettoiements, etc...);

- la localisation;

- l'année (fixée ou recommandée) et la périodicité;

- et la quotité.

L'intérêt primordial d'un tel document, même succinct, résulte de la nécessité pour le propriétaire (et pour ses descendants) de disposer d'un programme d'action précis, excluant les actions désordonnées ou pratiquées selon l'inspiration ou l'opportunité du moment, et garantissant:

- l'ordre et la continuité de vues, indispensables à la réalisation des objectifs préalablement fixés; 
- la répartition rationnelle des efforts dans le temps;

- l'absence de tout retard dans les interventions;

- l'économie de temps et de moyens,

et finalement l'obtention d'une forêt aussi homogène, aussi équilibrée et aussi proche que possible de son état de productivité maximum.

Il a surtout été question ci-dessus des forêts de rapport.

Je n'ajoute qu'un mot pour les forêts que le propriétaire désire conserver par exemple pour son agrément ou comme terrain de chasse: elles comportent, comme les autres, des arbres qui naissent, vieillissent et meurent; elles demandent également des exploitations et souvent des travaux; elles posent également le problème de leur pérennité et de leur régénération: même si l'on se désintéresse de la production ligneuse, l'on ne peut se passer d'un certain nombre de règles de gestion, même réduites à leur plus simple expression.

Il ne m'est pas possible, sous peine d'allonger hors de mesure le cadre de ces quelques notes, de passer ici en revue tous les cast particuliers de plans de gestion possibles. Pour les lecteurs de cette Revue intéressés par la question, un prochain article pourra donner quelques exemples applicables aux cas les plus usuels de forêts à traiter en futaie ou de taillis-sous-futaie à convertir en futaie. 